\title{
ORDEN DE LORENZ EN LA FAMILIA DE DISTRIBUCIONES GAMMA TRIPARAMÉTRICAS LORENZ ORDERING OF THREE PARAMETER GAMMA DISTRIBUTIONS
}

Héctor M. Ramos ${ }^{1}$

hector.ramos@uca.es

Miguel A. Sordo ${ }^{1}$

mangel.sordo@uca.es

Universidad de Cádiz

\section{Resumen}

El orden de Lorenz es una herramienta adecuada para comparar la desigualdad de dos distribuciones de rentas. En este artículo obtenemos una condición suficiente para que dos distribuciones sean comparables en el orden de Lorenz y aplicamos el resultado para ordenar la familia de distribuciones Gamma triparamétricas.

Palabras clave: Orden de Lorenz; Distribución Gamma.

\section{Abstract}

Lorenz ordering is an useful tool for comparing inequality of income distributions. In this note, we give a sufficient condition for this order and then apply it to order the three parameter family of Gamma distributions.

Keywords: Lorenz ordering; Gamma distribution.

${ }^{1}$ Departamento de Estadística e I.O., Facultad de CC. Económicas y Empresariales, Universidad de Cádiz, Duque de Nájera 8, 11002 Cádiz (España). 


\section{INTRODUCCIÓN}

El orden de Lorenz permite la comparación en desigualdad de dos distribuciones de renta. Sea $F_{X}(x)$ la función de distribución de una variable aleatoria $X$ no negativa y con media finita $\mu_{X}$. La curva de Lorenz, también llamada curva de concentración, correspondiente a $X$ se define (Gastwirth, 1971) como:

$$
L_{X}(p)=\frac{1}{\mu_{X}} \int_{0}^{p} F_{X}^{-1}(t) d t \quad 0 \leq p \leq 1
$$

Donde por $F_{X}^{-1}$ denotamos la inversa de $F_{X}$ definida por

$F_{X}^{-1}(p)=\inf \left\{x: F_{X}(x) \geq p\right\}, \quad p \in[0,1]$.

Si $X$ representa ingresos anuales, entonces $L_{X}(p)$ es la proporción del total de ingresos que corresponde a los individuos que se encuentran en el $100 p \%$ de ingresos más bajos. Un detallado estudio de la curva de Lorenz podemos encontrarlo en Gail y Gastwirth (1978), así como una relación precisa de sus propiedades en Dagum (1985). La curva de Lorenz nos permite definir el siguiente orden parcial $\left(\leq_{L}\right)$ sobre la clase de variables aleatorias no negativas:

$X \leq_{L} Y \Leftrightarrow L_{X}(p) \geq L_{Y}(p)$ para todo $0 \leq p \leq 1$. (2)

Si $X \leq_{L} Y$, entonces diremos que $X$ es menos desigual que $\mathrm{Y}$ en el sentido de Lorenz. Algunas referencias clásicas sobre el orden de Lorenz son Atkinson (1970), Dasgupta, Sen y Starrett (1973), Rothschild y Stiglitz (1973), Kakwani (1984) y Arnold (1987). De (1) y (2) se deduce fácilmente que el orden de Lorenz es invariante frente a transformaciones de escala; es decir, $X \leq_{L} Y$ si y sólo si N $a X \leq_{L} b Y$ para todo $a>0, b>0$. En

\section{INTRODUCTION}

Lorenz ordering is used to compare the amounts of inequality in two income distributions. Let $F_{X}(x)$ be the distribution function of a non-negative random variable $X$ with finite mean $\mu_{X}$, then the Lorenz curve, also called curve of concentration, corresponding to $X$ can be defined (Gastwirth, 1971) as:

$$
L_{X}(p)=\frac{1}{\mu_{X}} \int_{0}^{p} F_{X}^{-1}(t) d t \quad 0 \leq p \leq 1
$$

where we denote by $F_{X}^{-1}$ the inverse of $F_{X}$ defined by

$F_{X}^{-1}(p)=\inf \left\{x: F_{X}(x) \geq p\right\}, \quad p \in[0,1]$.

If $X$ represents annual income, $L_{X}(p)$ is the proportion of total income that accrues to individuals having the $100 \mathrm{p} \%$ lowest incomes. There is extensive discussion of the Lorenz curve in Gail and Gastwirth (1978) and a concise account of its properties in Dagum (1985). The Lorenz curve can be used to define a partial ordering (denoted $\leq_{L}$ ) on the class of non-negative random variables, as follows:

$$
X \leq_{L} Y \Leftrightarrow L_{X}(p) \geq L_{Y}(p) \text { for every } 0 \leq p \leq 1 \text {. (2) }
$$

If $X \leq_{L} Y$, then $X$ is said to exhibit less inequality in the Lorenz or relative Lorenz sense than $Y$. Some standard references for the Lorenz order are Atkinson (1970), Dasgupta, Sen and Starrett (1973), Rothschild and Stiglitz (1973), Kakwani (1984) and Arnold (1987). It is obvious from (1) and (2) that the Lorenz order is scale-invariant, that is, $X \leq_{L} Y$ if and only if $a X \leq_{L} b Y$ for all $a>0, b>0$. Arnold [1] shows that

$$
X+a \leq_{L} X \text {, for all } a>0 \text {, }
$$


Arnold [1] puede verse que

$$
X+a \leq_{L} X \text {, para todo } a>0,
$$

para toda variable aleatoria $X$ no negativa con media finita.

El orden de Lorenz nos permite establecer una comparación en desigualdad de forma absoluta, es decir, al margen de cualquier medida concreta de desigualdad que pudiera considerarse, las cuales, necesariamente, deben ser coherentes con dicho orden.

Desafortunadamente, no siempre dos variables aleatorias $X$ e $Y$ con distribuciones conocidas son susceptibles de ser comparadas en desigualdad en el sentido del orden de Lorenz. En este trabajo proponemos una condición suficiente para el orden de Lorenz cuyo cumplimiento es fácilmente contrastable. Esta condición suficiente será la que utilizaremos en la Sección 3 para la ordenación en desigualdad de la familia Gamma triparamétrica que puede ser usada como modelo probabilístico para la distribución de la renta.

Consideraremos los siguientes resultados previos (Shaked, 1982):

Definición 1 Sea $h(x)$ una función real definida en $I \subset \mathbf{R}$. El número de cambios de signo de $h$ en $I$ se define como sigue:

$$
S(h)=\sup S\left[h\left(x_{1}\right), h\left(x_{2}\right), \ldots . ., h\left(x_{m}\right)\right](4)
$$

donde $S\left[h\left(x_{1}\right), h\left(x_{2}\right), \ldots, h\left(x_{m}\right)\right]$ es el número de cambios de signo una vez eliminados los términos iguales a cero, y el supremo en (4) se extiende a todos los conjuntos $x_{1}<x_{2}<\ldots<x_{m} \quad\left(x_{i} \in I\right)$, $m<\infty$.

Necesitaremos el siguiente resultado. for every non-negative random variable $X$ with finite mean.

Lorenz ordering may be viewed as the maximal ranking generated by relative inequality measures, that is, if $X \leq_{L} Y$ then, whatever the measures of relative inequality one may choose, $Y$ must not be judged as less unequal that $X$ and conversely.

Unfortunately, for the two random variables $X$ and $Y$ with known distributions, it is sometimes not clear how to verify the relation $X \leq_{L} Y$. In this paper, we give a simple condition that ensure these orderings. This condition will be used in Section 3 for the ordering of the three parameter Gamma income distribution model. As in Shaked (1982), the following notation is used:

Definition 1 Let $h(x)$ be a real function defined in $I \subset \mathbf{R}$. The number of sign changes of $h$ in $I$ is defined by

$$
S(h)=\sup S\left[h\left(x_{1}\right), h\left(x_{2}\right), \ldots, h\left(x_{m}\right)\right]
$$

where $S\left[h\left(x_{1}\right), h\left(x_{2}\right), \ldots ., h\left(x_{m}\right)\right]$ is the number of sign changes of the indicated sequence, zero terms being discarded, and the supremum in (4) is extended over all sets $x_{1}<x_{2}<\ldots<x_{m} \quad\left(x_{i} \in I\right)$, $m<\infty$.

We require the following well known result. 
Teorema 2 Sean $X$ e $Y$ variables aleatorias continuas con igual media $\mu_{X}=\mu_{Y} \quad y$ sean $F \quad y \quad G$ sus correspondientes funciones de densidad. si $S(F-G)=1$ y la secuencia de signos es,-+ , entonces

$\int_{0}^{u} F^{-1}(t) d t \geq \int_{0}^{u} G^{-1}(t) d t$, para todo $0 \leq u \leq 1$.

(5)

Demostración. De acuerdo con lo asumido en el enunciado, tendremos que

$$
S\left(F^{-1}-G^{-1}\right)=1
$$

siendo la secuencia de signos,+- .

Por lo tanto, la integral

$$
\int_{0}^{u}\left[F^{-1}(t)-G^{-1}(t)\right] d t
$$

alcanza su menor valor cuando $u=1$.

Por otra parte, de la igual de medias se sigue que

$$
\int_{0}^{u}\left[F^{-1}(t)-G^{-1}(t)\right] d t \geq \int_{0}^{1}\left[F^{-1}(t)-G^{-1}(t)\right] d t=0,
$$
$y$, en consecuencia, se verifica (5).

\section{CONDICIONES SUFICIENTES PARA EL ORDEN DE LORENZ}

Utilizaremos el siguiente resultado de Arnold (1987).

Teorema 3 Sean $X$ and $Y$ variables aleatorias no negativas con medias finitas $\mu_{X}$ y $\mu_{Y}$, respectivamente, y sean $F y$ $G$ sus correspondientes funciones de distribución. Si $S\left(F\left(x \mu_{X}\right)-G\left(x \mu_{Y}\right)\right)=1$ $y$ la secuencia de signos es,-+ , entonces $X \leq_{L} Y$.
Theorem 2 Let $X$ and $Y$ be continuous random variables with equal means $\mu_{X}=\mu_{Y}$ and let $F$ and $G$ the corresponding densities. If $S(F-G)=1$ and the sign sequence is,-+ , then

$\int_{0}^{u} F^{-1}(t) d t \geq \int_{0}^{u} G^{-1}(t) d t$, for all $0 \leq u \leq 1$.

(5)

Proof. By the assumptions on $F$ and $G$ we have that

$S\left(F^{-1}-G^{-1}\right)=1$

with the sequence,+- .

Therefore, the integral

$\int_{0}^{u}\left[F^{-1}(t)-G^{-1}(t)\right] d t$ assumes its smallest value for $u=1$. From the equality of the means it follows that

$\int_{0}^{u}\left[F^{-1}(t)-G^{-1}(t)\right] d t \geq \int_{0}^{1}\left[F^{-1}(t)-G^{-1}(t)\right] d t=0$, and consequently (5) holds.

\section{SUFFICIENT CONDITIONS FOR LORENZ ORDERING}

The next theorem, due to Arnold (1987), will be used below.

Theorem 3 Let $X$ and $Y$ be nonnegative random variables with finite means $\mu_{X}$ and $\mu_{Y}$, respectively, and let $F$ and $G$ be the corresponding distribution functions. If $S\left(F\left(x \mu_{X}\right)-G\left(x \mu_{Y}\right)\right)=1$ and the sign sequence is,-+ , then $X \leq_{L} Y$. 
Demostración. Sean $F_{\frac{X}{\mu_{X}}}$ y $\frac{G_{Y}}{\mu_{Y}}$ las funciones de distribución de $\frac{X}{\mu_{X}}$ y $\frac{Y}{\mu_{Y}}$, respectivamente. Puesto que

$$
S\left(F_{\frac{X}{\mu_{X}}}(x)-G_{\frac{Y}{\mu_{Y}}}(x)\right)=S\left(F\left(x \mu_{X}\right)-G\left(x \mu_{Y}\right)\right)=1
$$

y la secuencia de signos es -,+, del Teorema 2 se sigue que $\frac{X}{\mu_{X}} \leq_{L} \frac{Y}{\mu_{Y}}$. Y ya que el orden de Lorenz es invariante frente a transformaciones de escala, tendremos que $X \leq_{L} Y$.

Basándonos en el Teorema 3, obtenemos en el siguiente corolario una condición suficiente para la comparación en desigualdad en el sentido de Lorenz de dos variables aleatorias absolutamente continuas.

Corolario 4 Sean $X$ e $Y$ variables aleatorias absolutamente continuas y no negativas, con medias finitas $\mu_{X} y \mu_{Y}, y$ soportes $\operatorname{supp}(X)$ y $\operatorname{supp}(Y)$, respectivamente, $y$ sean $f y g$ sus correspondientes funciones de densidad. Asumiremosque $\operatorname{supp}\left(\frac{X}{\mu_{X}}\right) \subseteq \operatorname{supp}\left(\frac{Y}{\mu_{Y}}\right)$. En estas condiciones, si $f\left(\mu_{X} x\right) / g\left(\mu_{Y} x\right)$ es unimodal para valores de $\boldsymbol{X}$ restringidos al $\operatorname{supp}\left(\frac{Y}{\mu_{Y}}\right)$, donde la moda es un supremo, entonces $X \leq_{L} Y$.

Demostración. Puesto que

$$
f_{\frac{X}{\mu_{X}}}(x)=\mu_{X} f\left(\mu_{X} x\right), \quad g_{\frac{Y}{\mu_{Y}}}(x)=\mu_{Y} g\left(\mu_{Y} x\right)
$$

Proof. Let $F_{\frac{X}{\mu_{X}}}$ and $\frac{G_{Y}}{\mu_{Y}}$ be the distribution functions of $\frac{X}{\mu_{X}}$ and $\frac{Y}{\mu_{Y}}$, respectively. Since

$$
S\left(F_{\frac{X}{\mu_{X}}}(x)-G_{\frac{Y}{\mu_{Y}}}(x)\right)=S\left(F\left(x \mu_{X}\right)-G\left(x \mu_{Y}\right)\right)=1
$$

and the sign sequence is,-+ , it follows from Theorem 2 that $\frac{X}{\mu_{X}} \leq_{L} \frac{Y}{\mu_{Y}}$. Since the Lorenz order is invariant under scale transformations we have that $X \leq_{L} Y$.

Based on Theorem 3 the next corollary gives a sufficient condition for the Lorenz comparison of two absolutely continuous random variables.

Corollary 4 Let $X$ and $Y$ be nonnegative and absolutely continuous random variables with finite means and supports $\operatorname{supp}(X)$ and $\operatorname{supp}(Y)$, respectively, and let $f$ and $g$ be the corresponding densities. Assume that supp $\left(\frac{X}{\mu_{X}}\right) \subseteq \quad \operatorname{supp} \quad\left(\frac{Y}{\mu_{Y}}\right)$. If $f\left(\mu_{X} x\right) / g\left(\mu_{Y} x\right)$ is unimodal for $X$ restricted to $\operatorname{supp}\left(\frac{Y}{\mu_{Y}}\right)$, where the mode is a supremum, then $X \leq_{L} Y$.

Proof. Since

$$
f_{\frac{X}{\mu_{X}}}(x)=\mu_{X} f\left(\mu_{X} x\right), \quad g_{\frac{Y}{\mu_{Y}}}(x)=\mu_{Y} g\left(\mu_{Y} x\right)
$$


y $f\left(\mu_{X} x\right) / g\left(\mu_{Y} x\right)$ es unimodal en $\operatorname{supp}\left(\frac{Y}{\mu_{Y}}\right)$, entonces también lo será $f_{\frac{X}{\mu_{X}}}(x) / g_{\frac{Y}{\mu_{Y}}}(x)$, siendo la moda un supremo. Por lo tanto,

$S\left({\frac{f}{\mu_{X}}}_{\mu_{X}}-g_{\frac{Y}{\mu_{Y}}}\right)=S\left(\frac{f_{\frac{X}{\mu_{X}}}}{g_{\frac{Y}{\mu_{Y}}}}-1\right) \leq 2$.

Como el orden estocástico no puede darse ya que $\frac{X}{\mu_{X}}$ y $\frac{Y}{\mu_{Y}}$ tienen la misma media, tendremos que $S\left(f_{\frac{X}{\mu_{X}}}-g_{\frac{Y}{\mu_{Y}}}\right)$ $=2$ siendo la secuencia de signos $-,+,-\quad y, \quad$ en consecuencia, $S\left(F_{\frac{X}{\mu_{X}}}-G_{\frac{Y}{\mu_{Y}}}\right)=1$ siendo la secuencia de signos,-+ . Finalmente, del Teorema 3 se sigue que $X \leq_{L} Y$.

Observación 5 Una condición suficiente para que $\mathrm{f} / \mathrm{g}$ sea unimodal es que $\mathrm{f} / \mathrm{g}$ sea log-concava (Keilson y Gerber, 1971).

Corolario 6 Sean $X$ e $Y$ variables aleatorias absolutamente continuas con medias finitas $y$ soportes respectivos $\operatorname{supp}(X)=(a, \infty) \quad y \quad \operatorname{supp}(Y)=(b, \infty)$, $a>0, \quad b \geq 0, y$ sean $f$ y $g$ sus correspondientes funciones de densidad. si $f\left(\mu_{X} x\right) / g\left(\mu_{Y} x\right)$ decrece en $X$ para $x \geq \frac{a}{\mu_{X}}$, entonces $X \leq_{L} Y$. and $f\left(\mu_{X} x\right) / g\left(\mu_{Y} x\right)$ is unimodal on $\operatorname{supp}\left(\frac{Y}{\mu_{Y}}\right)$, so is $f_{\frac{X}{\mu_{X}}}(x) / g_{\frac{Y}{\mu_{Y}}}(x)$, with the mode yielding a supremum. Hence

$$
S\left({\frac{f}{\mu_{X}}}_{\frac{\mu_{X}}{\mu_{Y}}}-g_{\frac{Y}{\mu_{Y}}}\right)=S\left(\frac{f_{X}}{g_{\frac{Y}{\mu_{X}}}}-1\right) \leq 2 .
$$

Since $\frac{X}{\mu_{X}}$ and $\frac{Y}{\mu_{Y}}$ have the same mean, ordinary stochastic order is not possible, so that $S\left(\frac{f_{X}}{\mu_{X}}-g_{\frac{Y}{\mu_{Y}}}\right)=2$ and the sign sequence is,,-+- . Then $S\left(F_{\frac{X}{\mu_{X}}}-G_{\frac{Y}{\mu_{Y}}}\right)=1$ and the sign sequence is,-+ . From theorem 3 it follows that $X \leq_{L} Y$.

Remark 5 A sufficient condition for $\mathrm{f} / \mathrm{g}$ to be unimodal is for f/g to be log-concave (Keilson and Gerber, 1971).

Corollary 6 Let $X$ and $Y$ be absolutely continuous random variables with finite means and supportssupp $(X)=(a, \infty)$ and $\operatorname{supp}(Y)=(b, \infty), \quad a>0, b \geq 0$, and let $f$ and $g$ be the corresponding densities. If $f\left(\mu_{X} x\right) / g\left(\mu_{Y} x\right)$ decreases in $x$ for $x \geq \frac{a}{\mu_{X}}$, then $X \leq_{L} Y$. 
Demostración. En primer lugar probaremos que $\frac{a}{\mu_{X}}>\frac{b}{\mu_{Y}}$. Consideremos, por reducción al absurdo, que $\operatorname{supp}\left(\frac{Y}{\mu_{Y}}\right) \subseteq \operatorname{supp}\left(\frac{X}{\mu_{X}}\right)$, es decir que $\frac{b}{\mu_{Y}} \geq \frac{a}{\mu_{X}}$. Entonces, teniendo en cuenta (6), definiremos

$$
\frac{g_{\frac{Y}{\mu_{Y}}}(x)}{{\frac{f_{X}}{\mu_{X}}(x)}(x)}=\left\{\begin{array}{ccc}
\frac{\mu_{Y}}{\mu_{X}} \frac{g\left(\mu_{Y} x\right)}{f\left(\mu_{X} x\right)} & \text { if } & x>\frac{b}{\mu_{Y}} \\
0 & \text { if } & \frac{a}{\mu_{X}}<x \leq \frac{b}{\mu_{Y}}
\end{array}\right.
$$

Puesto que $f\left(\mu_{X} x\right) / g\left(\mu_{Y} x\right)$ es decreciente para $x \geq \frac{a}{\mu_{x}}$, tendremos que $g\left(\mu_{Y} x\right) / f\left(\mu_{X} x\right)$ es creciente para $x>\frac{b}{\mu_{Y}}$. En consecuencia,

$S\left(g_{\frac{Y}{\mu_{Y}}}-f_{\frac{X}{\mu_{X}}}\right)=S\left(\frac{g_{\frac{Y}{\mu_{Y}}}}{f_{\frac{X}{\mu_{X}}}}-1\right) \leq 1$

lo que no es posible ya que $\frac{Y}{\mu_{Y}}$ y $\frac{X}{\mu_{X}}$ tienen la misma media. Por tanto, se cumple que $\operatorname{supp}\left(\frac{X}{\mu_{X}}\right) \subset \operatorname{supp}\left(\frac{Y}{\mu_{Y}}\right)$ ' es decir, $\frac{a}{\mu_{X}}>\frac{b}{\mu_{Y}}$. Finalmente, puesto que $f\left(\mu_{X} x\right) / g\left(\mu_{Y} x\right)$ es decreciente para $x \geq a / \mu_{X}$, tenemos que $f\left(\mu_{X} x\right) / g\left(\mu_{Y} x\right)$ es unimodal para $x$ restringido a $\operatorname{supp}\left(\frac{Y}{\mu_{Y}}\right)$. Finalmente, aplicando el Corolario 4, el resultado queda demostrado.
Proof. First, we prove that $\frac{a}{\mu_{X}}>\frac{b}{\mu_{Y}}$. Assume, by the way of contradiction, that $\operatorname{supp}\left(\frac{Y}{\mu_{Y}}\right) \subseteq \operatorname{supp}\left(\frac{X}{\mu_{X}}\right)$, i.e. that $\frac{b}{\mu_{Y}} \geq \frac{a}{\mu_{X}}$. Then, using (6) we define

$$
\frac{g_{\frac{Y}{\mu_{Y}}}(x)}{f_{\frac{X}{\mu_{X}}}(x)}=\left\{\begin{array}{cccc}
\frac{\mu_{Y}}{\mu_{X}} \frac{g\left(\mu_{Y} x\right)}{f\left(\mu_{X} x\right)} & \text { if } & x>\frac{b}{\mu_{Y}} \\
0 & \text { if } & \frac{a}{\mu_{X}}<x \leq \frac{b}{\mu_{Y}}
\end{array}\right.
$$

Since $f\left(\mu_{X} x\right) / g\left(\mu_{Y} x\right)$ decreases in $x$ for $x \geq \frac{a}{\mu_{X}}$, it follows that $g\left(\mu_{Y} x\right) / f\left(\mu_{X} x\right)$ increases in $x$ for $x>\frac{b}{\mu_{Y}}$. Hence $S\left(g_{\frac{Y}{\mu_{Y}}}-f_{\frac{X}{\mu_{X}}}\right)=S\left(\frac{g_{\frac{Y}{\mu_{Y}}}}{f_{\frac{X}{\mu_{X}}}}-1\right) \leq 1$ which is not possible because $\frac{Y}{\mu_{Y}}$ and $\frac{X}{\mu_{X}}$ have the same mean. Thus, it follows that $\operatorname{supp}\left(\frac{X}{\mu_{X}}\right) \subset \operatorname{supp}\left(\frac{Y}{\mu_{Y}}\right)$, i.e. $\frac{a}{\mu_{X}}>\frac{b}{\mu_{Y}}$. Again, since $f\left(\mu_{X} x\right) / g\left(\mu_{Y} x\right)$ decreases in $x$ for $x \geq a / \mu_{X}$, we have that $f\left(\mu_{X} x\right) / g\left(\mu_{Y} x\right)$ is unimodal for $x$ restricted to $\operatorname{supp}\left(\frac{Y}{\mu_{Y}}\right)$. The result follows by applying Corollary 4. 


\section{APLICACIÓN}

En esta sección aplicaremos nuestro anterior resultado (Corolario 6) para la ordenación de la familia de distribuciones Gamma triparamétricas.

\subsection{Orden de Lorenz en la familia de distribuciones Gamma triparamétricas}

\section{APPLICATION}

\subsection{Lorenz ordering of three parameter Gamma distributions}

Let $X$ be the three-parameter Gamma distribution with density function

Sea $X$ la distribución Gamma triparamétrica cuya función de densidad es

$$
f_{X}(x)=\frac{\beta^{-\alpha}(x-\theta)^{\alpha-1} e^{-\left(\frac{x-\theta}{\beta}\right)}}{\Gamma(\alpha)}, \theta \geq 0, \alpha>0, \beta>0, x>\theta .
$$

Puesto que el orden de Lorenz es invariante frente a transformaciones de escala, podemos considerar, sin pérdida alguna de generalidad, que el parámetro de escala $\beta$ es igual a 1 por lo que
Since the Lorenz order is invariant under scale changes, the scale parameter $\beta$ can be set equal to 1 without loss of generality. This gives

$$
f_{X}(x)=\frac{(x-\theta)^{\alpha-1} e^{-(x-\theta)}}{\Gamma(\alpha)}, \alpha>0, \theta \geq 0, x>\theta .
$$

Llamaremos $G(\theta, \alpha)$. a la correspondiente función de distribución. Arnold, Brockett, Robertson y Shu (1987) consideran $X_{1} \sim G\left(\theta, \alpha_{1}\right)$ y $X_{2} \sim G\left(\theta, \alpha_{2}\right)$ ( $\theta$ fijo) y demuestran que $X_{2} \leq_{L} X_{1}$ para $\alpha_{1} \leq \alpha_{2}$. Por otra parte, si consideramos $X_{1} \sim G\left(\theta_{1}, \alpha\right)$ y $X_{2} \sim G\left(\theta_{2}, \alpha\right) \alpha$ (fijo) puede verse que $X_{2} \leq_{L} X_{1}$ para $\theta_{1} \leq \theta_{2}$. En efecto, puesto que $X_{2}=X_{1}+\left(\theta_{2}-\theta_{1}\right)$, con $\theta_{2}-\theta_{1}>0$, se sigue de (3) que $X_{2} \leq_{L} X_{1}$. Estos resultados pueden ser extendidos para $X_{1} \sim G\left(\theta_{1}, \alpha_{1}\right)$ y $X_{2} \sim G\left(\theta_{2}, \alpha_{2}\right)$. Aplicando la transitividad del orden de Lorenz y los anteriores resultados, es fácil probar que si $\alpha_{1} \leq \alpha_{2}$ y $\theta_{1} \leq \theta_{2}$, entonces $X_{2} \leq_{L} X_{1}$. Sin embargo, no sabemos qué ocurre cuando $\alpha_{1}<\alpha_{2}$ y $\theta_{1}>\theta_{2}$. Esta cuestión nos conduce al siguiente resultado.
The corresponding distribution will be denoted by $G(\theta, \alpha)$. Arnold, Brockett, Robertson and Shu (1987) consider $X_{1} \sim G\left(\theta, \alpha_{1}\right)$ and $X_{2} \sim G\left(\theta, \alpha_{2}\right)$ ( $\theta$ fixed $)$ and prove that $X_{2} \leq_{L} X_{1}$ for $\alpha_{1} \leq \alpha_{2}$. On the other hand, if we consider $X_{1} \sim G\left(\theta_{1}, \alpha\right)$, and $X_{2} \sim G\left(\theta_{2}, \alpha\right) \quad(\alpha$ fixed) it can be shown that $X_{2} \leq_{L} X_{1}$ for $\theta_{1} \leq \theta_{2}$. In fact, since $X_{2}=X_{1}+\left(\theta_{2}-\theta_{1}\right)$, with $\theta_{2}-\theta_{1}>0$, from (3) it follows that $X_{2} \leq_{L} X_{1}$. These results can be completed considering $X_{1} \sim G\left(\theta_{1}, \alpha_{1}\right)$ and $X_{2} \sim G\left(\theta_{2}, \alpha_{2}\right)$. Using the transitivity of the Lorenz order and the above results, it is easy to prove that if $\alpha_{1} \leq \alpha_{2}$ and $\theta_{1} \leq \theta_{2}$ then $X_{2} \leq_{L} X_{1}$. However, what happen if $\alpha_{1}<\alpha_{2}$ and $\theta_{1}>\theta_{2}$ ? This leads to the following result. 
Teorema 7 Si $X_{1} \sim G\left(\theta_{1}, \alpha_{1}\right)\left(\alpha_{1} \leq 1\right)$ y $X_{2} \sim G\left(\theta_{2}, \alpha_{2}\right)$, con $\theta_{1}-\theta_{2}>\alpha_{2}-\alpha_{1}>0$, entonces $X_{1} \leq_{L} X_{2}$.

Demostración. En primer lugar observemos que la relación

$$
\theta_{1}-\theta_{2}>\alpha_{2}-\alpha_{1}>0
$$

es posible únicamente si $\alpha_{1}<\alpha_{2}$ y $\theta_{1}>\theta_{2}$. Teniendo en cuenta que la función de densidad de $X \sim G(\theta, \alpha)$ es (8) y que $\mu=E[X]=\alpha+\theta$, tendremos que

$$
\begin{gathered}
\frac{f_{1}\left(\mu_{1} x\right)}{f_{2}\left(\mu_{2} x\right)}=k \cdot\left[\frac{\left(\alpha_{1}+\theta_{1}\right) x-\theta_{1}}{\left(\alpha_{2}+\theta_{2}\right) x-\theta_{2}}\right]^{\alpha_{1}-1} \cdot\left[\frac{1}{\left(\alpha_{2}+\theta_{2}\right) x-\theta_{2}}\right]^{\alpha_{2}-\alpha_{1}} . \\
\cdot \exp \left[\left(\alpha_{2}-\alpha_{1}+\theta_{2}-\theta_{1}\right) x\right] \\
\text { Donde/were, } k=\frac{\Gamma\left(\alpha_{2}\right)}{\Gamma\left(\alpha_{1}\right)} \cdot \exp \left(\theta_{1}-\theta_{2}\right)>0 .
\end{gathered}
$$

De (9) se sigue claramente que $\exp \left[\left(\alpha_{2}-\alpha_{1}+\theta_{2}-\theta_{1}\right) x\right]$ es decreciente en $x$. Supongamos ahora que $x \geq \theta_{1} / \mu_{1}=\theta_{1} /\left[\alpha_{1}+\theta_{1}\right]$. Puesto que $\alpha_{1}<\alpha_{2}$, se deduce que

$\left[\frac{1}{\left(\alpha_{2}+\theta_{2}\right) x-\theta_{2}}\right]^{\alpha_{2}-\alpha_{1}}$

es también decreciente en $x$ para $x \geq \theta_{2} /\left[\alpha_{2}+\theta_{2}\right]$. De (9) se sigue que $\frac{\theta_{1}}{\alpha_{1}+\theta_{1}}>\frac{\theta_{2}}{\alpha_{2}+\theta_{2}}$.

Por lo tanto, (10) es decreciente en $x$ para $x \geq \theta_{1} / \mu_{1}$. Finalmente, llamando $h(x)=\left[\frac{\left(\alpha_{1}+\theta_{1}\right) x-\theta_{1}}{\left(\alpha_{2}+\theta_{2}\right) x-\theta_{2}}\right]^{\alpha_{1}-1}, \alpha_{1}<1$,
From (9) we clearly have that $\exp \left[\left(\alpha_{2}-\alpha_{1}+\theta_{2}-\theta_{1}\right) x\right]$ decreases in $x$. Now, suppose $x \geq \theta_{1} / \mu_{1}=\theta_{1} /\left[\alpha_{1}+\theta_{1}\right]$. Since $\alpha_{1}<\alpha_{2}$, it follows that

$\left[\frac{1}{\left(\alpha_{2}+\theta_{2}\right) x-\theta_{2}}\right]^{\alpha_{2}-\alpha_{1}}$

is also decreasing in $x$ for $x \geq \theta_{2} /\left[\alpha_{2}+\theta_{2}\right]$. From (9) it follows that $\frac{\theta_{1}}{\alpha_{1}+\theta_{1}}>\frac{\theta_{2}}{\alpha_{2}+\theta_{2}}$.

Thus, (10) decreases in $x$ for $x \geq \theta_{1} / \mu_{1}$. Finally, denoting $h(x)=\left[\frac{\left(\alpha_{1}+\theta_{1}\right) x-\theta_{1}}{\left(\alpha_{2}+\theta_{2}\right) x-\theta_{2}}\right]^{\alpha_{1}-1}, \alpha_{1}<1$, 
se demuestra a partir de (9) que $h^{\prime}(x) \leq 0$ si y sólo si $\alpha_{2} \theta_{1}>\alpha_{1} \theta_{2}$. Por consiguiente, la razón $f_{1}\left(\mu_{1} x\right) / f_{2}\left(\mu_{2} x\right)$ es decreciente para $x \geq \theta_{1} / \mu_{1}$. Por tanto, aplicando el Corolario 6, el teorema queda demostrado. it can be proven that $h^{\prime}(x) \leq 0$ if and only if $\alpha_{2} \theta_{1}>\alpha_{1} \theta_{2}$, which follows from (9). Therefore, the assumption implies that the ratio $f_{1}\left(\mu_{1} x\right) / f_{2}\left(\mu_{2} x\right)$ is decreasing for $x \geq \theta_{1} / \mu_{1}$. Thus, applying Corollary 6 , the proof is complete.

\section{BIBLIOGRAFÍA/REFERENCES}

Arnold, B.C. (1987). Majorization and the Lorenz Order: A brief introduction. Berlin: SpringerVerlag.

Arnold, B.C., Brockett, P.L., Robertson, C.A. y Shu, B. (1987). Generating ordered families of Lorenz curves by strongly unimodal distributions. Journal of Business and Economic Statistics, 5, 305-308.

Atkinson, B. (1970). On the measurement of inequality. Journal of Economic Theory, 2, 244-263.

Dagum, C. (1985). Lorenz Curve, encyclopedia of statistical sciencies (5, pp. 156-161). S. Kotz, N.L. Johnson \& C.B. Read (Eds.). New York: Wiley.

Dasgupta, P., Sen, A.K. y Starrett, D. (1973). Notes on the measurement of inequality. Journal of Economic Theory, 6, 180-187.

Gail, M.H. y Gastwirth,J.L. (1978). A scale-free goodness-of-fit test for the exponential distribution based on the Lorenz curve. Journal of the American Statistical Association, 73, 786-793.

Gastwirth, J.L. (1971). A general definition of the Lorenz curve. Econometrica, 39, 1037-1039.

Kakwani, N. (1984). Welfare ranking of income distributions. Advances in Econometrics, 3, 191213.

Keilson, J. y Gerber, H. (1971). Some result for discrete unimodality. Journal of the American Statistical Association, 66, 386-389.

Rothschild, M. y Stiglitz, J.E. (1973). Some further results on the measurement of inequality. Journal of Economic Theory, 6, 188-204.

Shaked, M. (1982). Dispersive ordering of distributions. Journal of Applied Probability, 19, 310-320. 